\title{
Norois
}

Environnement, aménagement, société

$196 \mid 2005 / 3$

Géographie du littoral : approche géomatique

\section{Verger (F.) - Marais maritimes et estuaires du littoral français}

Paris, Belin, 2005, 335 p.

Lydie Goeldner-Gianella

\section{OpenEdition}

Journals

Édition électronique

URL : http://journals.openedition.org/norois/475

DOI : $10.4000 /$ norois. 475

ISBN : 978-2-7535-1544-4

ISSN : $1760-8546$

Éditeur

Presses universitaires de Rennes

Édition imprimée

Date de publication : 1 septembre 2005

Pagination : 138-139

ISBN : 978-2-7535-0218-5

ISSN : 0029-182X

Référence électronique

Lydie Goeldner-Gianella, «Verger (F.) - Marais maritimes et estuaires du littoral français », Norois [En ligne], 196 | 2005/3, mis en ligne le 15 décembre 2008, consulté le 23 septembre 2020. URL : http:// journals.openedition.org/norois/475; DOI : https://doi.org/10.4000/norois.475

Ce document a été généré automatiquement le 23 septembre 2020

(c) Tous droits réservés 


\section{Verger (F.) - Marais maritimes et estuaires du littoral français}

Paris, Belin, 2005, 335 p.

Lydie Goeldner-Gianella

\section{RÉFÉRENCE}

Verger (F.), 2005. - Marais maritimes et estuaires du littoral français, Belin, Paris, 335 p. 
1 Après deux réimpressions de l'ouvrage Marais et wadden du littoral français, initialement paru en 1968, Fernand Verger nous livre une édition entièrement renouvelée de sa thèse de géographie physique, qui s'intitule désormais Marais maritimes et estuaires du littoral français. L'ouvrage se scinde en deux parties, l'une consacrée à une présentation générale des estuaires, wadden et marais maritimes et l'autre, à l'analyse régionale de chacun des grands marais et estuaires de notre littoral, à travers onze chapitres promenant le lecteur du bassin d'Arcachon à la plaine maritime flamande (les onze secteurs présentés sont : le bassin d'Arcachon et ses rivages, les marais et l'estuaire girondins, les marais et pertuis charentais, le marais Poitevin et l'anse de l'Aiguillon, le marais Breton et la

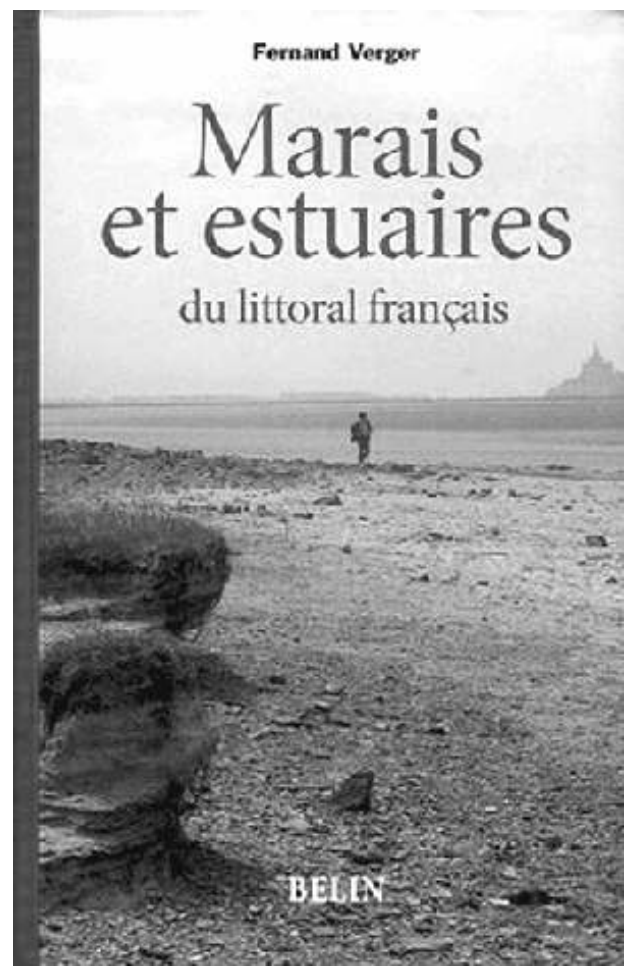
baie de Bourgneuf, les marais et l'estuaire de la Loire, le marais de Dol et la baie du Mont-Saint-Michel, le marais de Carentan et la baie des Veys, les marais et l'estuaire de la Seine, les marais et estuaires picards, la plaine maritime flamande).

2 Cet ouvrage fait d'emblée une forte impression, tant son iconographie, tout en couleurs, est superbe et variée. Pour la réalisation graphique de ses cartes de synthèse et nombreux schémas explicatifs, Fernand Verger a fait appel à Raymond Ghirardi, ingénieur cartographe à qui on devait déjà les excellentes illustrations de l'Atlas de Géographie de l'Espace (Verger [dir.], 1997, Belin). À cela s'ajoutent d'innombrables photographies, prises pour la plupart par l'auteur qui a arpenté ou même survolé les sites qu'il nous décrit. Judicieusement choisies, elles nous font mieux voir les paysages de marais (souvent photographiés depuis les hauteurs voisines), leurs formes et les processus qui y sont à l'œuvre. Des comparaisons de photographies aériennes, images satellites et cartes anciennes sont tout aussi instructives.

La particularité des régions de marais, nous dit Fernand Verger, est que « la géographie physique, domaine central $[\mathrm{du}]$ volume, [y] reconnaît l'action humaine comme agent majeur ». Et c'est l'une des raisons de la réécriture de l'ouvrage. Certes, les milieux physiques de marais et d'estuaires ont évolué de façon naturelle en 40 ans - et Fernand Verger évoque notamment la tempête de décembre 1999 ou les effets à attendre de l'élévation du niveau de la mer -, mais ils ont aussi évolué sous l'effet d'une intervention anthropique de grande ampleur dans la seconde moitié $\mathrm{du} \mathrm{xx}^{\mathrm{e}}$ siècle, avec la multiplication des endiguements, remblaiements et barrages et l'intensification des dragages et du drainage. Par ailleurs, la perception sociale des marais et des wadden s'est, elle aussi, largement modifiée. L'on est ainsi passé de l'aménagement au « ménagement » de ces milieux et à une gestion de plus en plus collective et tournée vers le développement durable. De ce fait, l'auteur a complètement réactualisé ses problématiques : de Natura 2000 aux premières dépoldérisations, du rétablissement du 
caractère maritime du Mont-Saint-Michel aux mesures de compensation des grands aménagements portuaires, de la Loi Littoral aux syndicats mixtes de gestion des marais, Fernand Verger évoque les principaux aspects de la protection, de la gestion et de la restauration de ces milieux. Son chapitre 3 constitue une excellente synthèse de l'évolution de la législation de protection des zones humides. L'intérêt de ce géographe physicien pour la protection et la gestion des marais se voit d'ailleurs à son engagement comme conseiller scientifique du Conservatoire du Littoral ou président de la commission scientifique de la Mission Mont-Saint-Michel.

Cette part plus grande faite au temps présent et à l'Homme explique que le texte intial ait été réduit, tant sur le plan bibliographique que scientifique. Il reste néanmoins deux cents références empruntées aux grands maîtres de la géographie physique française et nord-européenne, comme à leurs successeurs, à des gestionnaires de marais ou à des spécialistes d'autres disciplines. Par ailleurs, les précisions géomorphologiques, sédimentologiques, stratigraphiques ou pédologiques de l'édition originale, ont été synthétisées et regroupées dans les quatre premiers chapitres de l'ouvrage. Le chapitre introductif est ainsi consacré aux caractéristiques de la marée et aux niveaux de référence hydrographiques et terrestres, de même qu'à l'élévation passée et présente du niveau de la mer. Le second chapitre évoque les matériaux du colmatage, là aussi de façon plus synthétique : sont analysés la nature et le comportement des sédiments, les difficultés et méthodes des bilans sédimentaires, l'évolution pédologique des schorres et des marais endigués. Un troisième chapitre aborde plus en détail les éléments faunistiques et floristiques des différents milieux et ce, selon un transect allant de la basse slikke au fond des marais endigués, en passant par les digues et le réseau hydrographique artificiel. L'auteur s'étend plus longuement et à juste titre sur l'avifaune migratrice. Enfin, une géomorphologie descriptive nous fait entrevoir la richesse des formes présentes dans les marais endigués et ce à différentes échelles, de leurs pentes générales à des microformes telles que les mottureaux. La description des wadden s'achève par une utile classification géomorphologique, fondée sur l'arrangement régional de leurs différentes formes élémentaires. Cet effort général de synthèse s'est toutefois fait en accordant une place plus grande aux estuaires, qui abritent de vastes étendues de wadden. Les pages consacrées à l'estuaire ligérien fournissent par exemple moins d'éléments sur les processus sédimentaires locaux, mais s'attardent davantage sur des aspects contemporains plus importants - comme les modifications de l'estuaire, de ses marais et de ses îles, du fait de l'approfondissement de son chenal ou du remblaiement progressif de ses berges - ou donnent un excellent aperçu de la géographie physique et humaine des marais de la Grande Brière, non évoquée antérieurement.

5 La contraction du contenu initial de Marais et wadden a également permis de le rendre plus accessible aux géographes non-spécialistes de ces milieux, mais aussi à un plus vaste public. La diffusion de l'ouvrage en sera assurément élargie, ce qui tient non seulement à la clarté de l'écriture et à la richesse de l'iconographie, mais aussi à la présence d'un lexique final regorgeant de termes vernaculaires et à des schémas de synthèse particulièrement didactiques - auparavant publiés dans différentes revues de géographie - et dont Fernand Verger est passé maître (bloc-diagramme composite illustrant la définition des principales formes des marais et des wadden, blocdiagramme illustrant l'évolution de la perception sociale des côtes à vasières, schéma des milieux et de leur utilisation dans la baie du Mont-Saint-Michel ou l'Anse de l'Aiguillon...). Au final, cet ouvrage remarquable nous semble lancer un double défi : 
celui de donner à tout géographe, mais aussi à un plus large public, l'envie de lire une géographie physique modernisée, tout en incitant chaque lecteur à revisiter en parallèle la littérature française, dont des extraits fort bien choisis émaillent l'entrée de chaque chapitre.

\section{AUTEUR}

\section{LYDIE GOELDNER-GIANELLA}

UMR 8586 CNRS PRODIG, Université Paris 1 Panthéon-Sorbonne, Institut de Géographie - 191, rue St-Jacques, 75005 Paris cedex, France, Lydie.Goeldner-Gianella@univ-paris1.fr 